\title{
POPULATION GENETICS OF A "COLONISING" LIZARD: NATURAL SELECTION FOR ALLOZYME MORPHS IN ANOLIS GRAHAMI
}

\author{
CHARLES E. TAYLOR \\ Department of Biology, University of California, Riverside, CA \\ and \\ GEORGE C. GORMAN \\ Department of Biology, University of California, Los Angeles, CA

\begin{abstract}
SUMMARY
Allozyme frequencies of Anolis grahami from Bermuda were compared to those from Jamaica. These lizards were introduced into Bermuda from Jamaica in 1905. The magnitude of the genetic changes were consistent with the hypothesis that these changes were produced by random genetic drift. The changes for the separate alleles were, however, more heterogeneous than expected if caused by random drift alone. It is concluded that not all the alleles examined have been selectively neutral.
\end{abstract}

Received 4.iii.75

\section{INTRODUCTION}

Electrophoretrc techniques are being routinely utilised to analyse levels of genetic variation within and between populations. Of great interest to population geneticists and evolutionary biologists is whether differences observed between populations reflect merely random events (random genetic drift) or indicate natural selection. Lewontin and Krakauer (1973) recently showed that for neutral alleles a simple transform of the inbreeding coefficient is distributed approximately as chi-square. Thus, if independent estimates of the inbreeding coefficients for alleles segregating in the same populations are calculated, then either this transform of the inbreeding coefficient has a chi-square distribution or else the alleles are not all neutral. This test has been used to infer selection for allozymes in Drosophila pseudoobscura (Lewontin, 1974), burrowing rodents (Nevo, 1973), in some populations of island lizards (Gorman et al., 1975) and for olive fruit flies, Dacus oleae (Lewontin and Krakauer, 1973). While criticised for use in comparing many different populations, this method is appropriate for the comparisons made below ( $R$. G. Lewontin, personal communication).

In this study we compare the distribution of inbreeding coefficients for allozyme morphs in the lizard Anolis grahami collected on Bermuda and Jamaica. This analysis demonstrates that not all the allozyme morphs examined from these populations are likely to be neutral.

Anolis lizards are widely distributed in the warmer parts of the Americas, and are particularly successful as island colonists. There is hardly an island in the Caribbean that lacks anoles, and almost each island bank has an anole fauna comprised primarily of endemic forms. Bermuda (or, more 
correctly, the Bermuda island) is a small, rather remote landmass, lying 600 miles east of North Carolina in the open Atlantic at a latitude of about $32^{\circ} \mathrm{N}$. Despite its relatively northerly latitude, it is warmed by the Gulf stream and supports a subtropical and warm temperate vegetation (Wingate, 1965).

Bermuda is not easily colonised by non-flying terrestrial vertebrates, and its native reptilian fauna consists of a single lizard, an endemic species of skink. However, several amphibians and reptiles from more tropical latitudes have been introduced by man, and are thriving.

Bermuda is presently inhabited by three species of Anolis lizards from three different Caribbean islands. Two of these species have unknown and perhaps accidental "colonisation" histories. Anolis leachi, a native of Antigua, was first discovered in 1956 and has a very limited range in central Bermuda; Anolis extremus, a native of Barbados, was first recorded in 1953 and is restricted to the far western part of Bermuda (Wingate, 1965). The Anolis grahami situation is much more interesting for a population genetics analysis because the initial introduction and subsequent spread of the lizard (to all parts of Bermuda) has been well documented.

Anolis grahami is a species endemic to the island of Jamaica. In September 1905, the Director of Agriculture for Bermuda released 26 males and 45 females that had been collected in the area of Kingston. Thus we know the size of the initial population. Since female Anolis can store sperm (Fox, 1963), some of the introduced females were presumably carrying genetic information from males that were not introduced. Thus, in round numbers, we can consider our colonisation to have consisted of approximately 100 individuals. Although we lack detailed data on the demography of this small lizard, it is not unreasonable to estimate that the population turns over approximately on an annual basis, so we also have a good estimate of the number of generations since the introduction.

Anolis grahami dispersed quickly on Bermuda. Two were seen a mile from the site of the introduction within 6 weeks. Island-wide distribution was realised sometimes between 1935 and 1945. The detailed documentation of dispersal can be found in Wingate's publication. Wingate suggested that because Anolis was a novelty on Bermuda, dispersion may have been aided by intentional capture and release in gardens. Today, this species is abundant, and individuals must number 100,000 or more (Bermuda is 21 square miles, or 13,400 acres; $A$. grahami averages well over seven individuals per acre).

Bermuda provides a novel selective regime for a Jamaican lizard. Despite its mild climate, Bermuda is far more seasonal than Jamaica $\left(18^{\circ} \mathrm{N}\right)$. Licht and Gorman (1970) demonstrated that $A$. grahami is highly seasonally reproductive on Bermuda, much less so at Kingston, Jamaica. Furthermore, on Jamaica, A. grahami often occurs sympatrically with up to five additional native species of Anolis; whereas on Bermuda it was the exclusive arboreal lizard for a number of years, is now sympatric with only one other anole at any given locality ( $A$. leachi and $A$. extremus do not overlap), and is still the only anole throughout much of its range. This could provide ample room for " ecological release", and indeed, perch preferences seem much broader on Bermuda than Jamaica. Thus we might expect that if allozymes are not all neutral, the effects of selection might demonstrate themselves at the level of biochemical gene products. 


\section{MAterials, methods AND ELEGTROPHORETIC RESUlts}

In November 1973 a series of 43 Anolis grahami from the eastern, central and western parts of Bermuda was shipped to UCLA. In December 1973 a series of 38 Anolis grahami from the area of Kingston, Jamaica (specifically, Hope Gardens, and the nearby campus of the University of the West Indies),

TABLE 1

Allele frequencies and estimated inbreeding coefficients for allozymes of Anolis grahami. An asterisk indicates that an inbreeding coefficient was not estimated because the allele frequency was too low.

I. Polymorphic loci

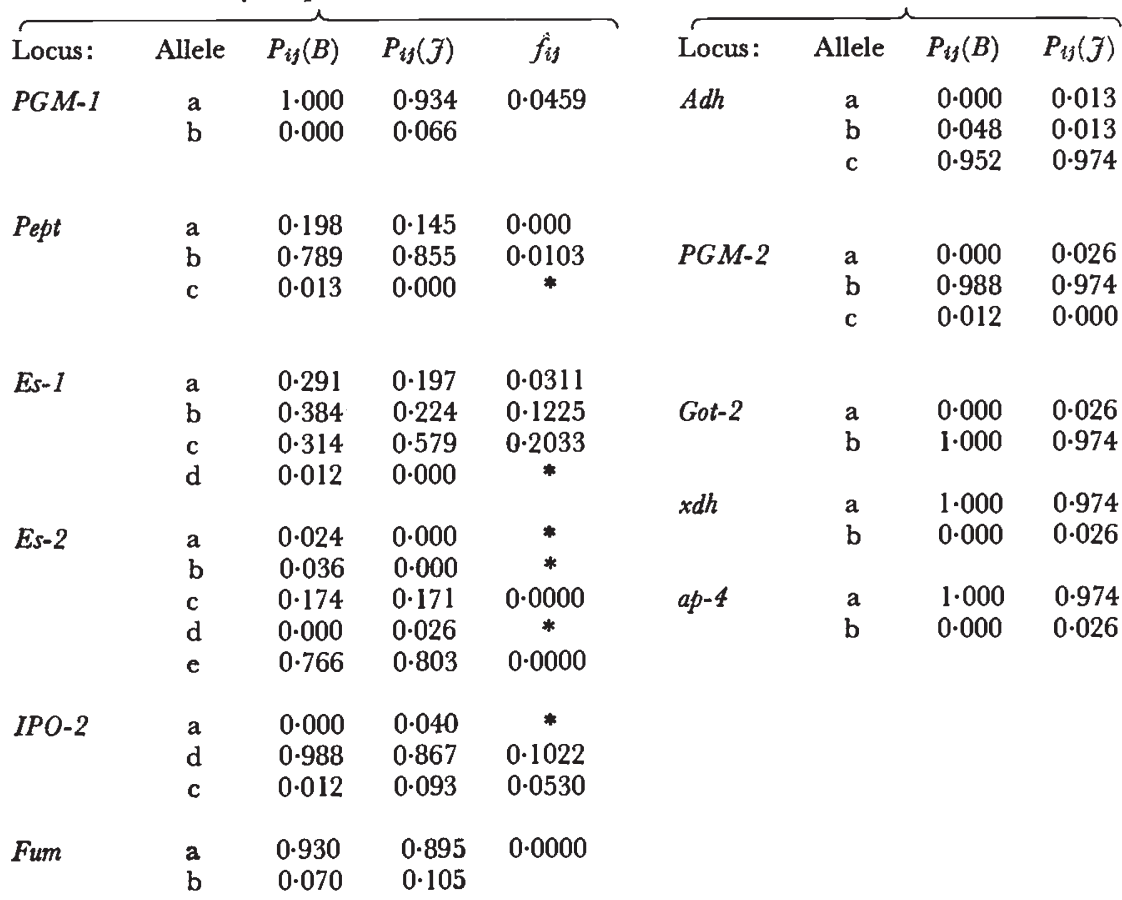

The following loci were fixed identically in both populations; $P g m-3, M d h-1, M d h-2$, Ldh-1, Ldh-2, Gol-1, 6-Pgd, Pgi, Gp-1, Gp-2, Gp-3, Ipo-1

Abbreviations utilised are: Gp-, general proteins stained with Buffalo Black; Es-, esterases; $\alpha$-Gpd, $\alpha$-glycerophosphate dehydrogenase; Adh, alcohol dehydrogenase; Pgm", phosphoglucomutases; Pept, peptidase; Got-, glutamate oxalate transaminases; Xdh, Xanthine dehydrogenase; Ipo-, indophenol oxidases; Fum, fumerase; Mdh-, malate dehydrogenases; Ldh-, lactate dehydrogenases; 6-Pgd, 6-phosphogluconate dehydrogenase; Pgi, phosphoglucose isomerase.

Alleles are designated alphabetically, with " a " being the least anodal.

was shipped to UCLA. The lizards arrived alive and were frozen at $-68^{\circ} \mathrm{C}$ until they were processed. Electrophoresis was carried out as outlined in our recent papers (Yang et al., 1974; Gorman et al., 1975).

A total of 24 loci was scored for each individual lizard. Of these, 12 were fixed identically in both populations and 12 were variable in one or both populations. Table 1 summarises the allele frequencies for the variable 
loci. Table 2 summarises levels of variability in the populations. It should be noted that the Bermuda samples were originally treated as separate subsamples by locality but were pooled because there were no significant differences between the subsamples.

The percentage of variable (polymorphic) loci was much higher in the parental Jamaican population ( 50 per cent $v .29$ per cent), but average individual heterozygosity was not strikingly different $(7 \cdot 8$ per cent $v$. 6.4 per cent on Jamaica and Bermuda, respectively). There were nine alleles

TABLE 2

Summary of variability in Anolis grahami from Bermuda and Jamaica

$\begin{array}{lcc}\text { Sample size } & 38 & 43 \\ \text { Number of loci } & 24 & 24 \\ \text { Number of variable loci } & 12 & 7 \\ \text { Percentage of variable loci } & 50 & 29 \\ \text { Percentage of heterozygosity } & 7 \cdot 8 & 6.4 \\ \text { Alleles/locus } & 1 \cdot 75 & 1.50\end{array}$

at nine loci that were present in Jamaica and absent on Bermuda. These were all relatively rare alleles, with frequencies ranging from 0.013 to 0.066 . There were also five alleles at four loci that were present in Bermuda and absent in Jamaica. These were ever rarer, ranging from 0.012 to 0.036 . These alleles may have been present on Jamaica but not found because of their rarity. With only one exception (Esterase-1) the most frequent allele at each locus was the same in both populations.

\section{Genetic interpretation AND discussion}

Under the assumption that Kingston, Jamaica, Anolis grahami are under the same selective regime that prevailed in 1905, random genetic drift would account for any differences between our 1973 sample and one that would have been made in 1905 . The Jamaican populations have been very large all throughout this time period, and we would expect the effects of drift to be minimal. If random genetic drifts were responsible for the differences between allele frequencies on Jamaica $\left(P_{i j}(J)\right)$ and Bermuda $\left(P_{i j}(B)\right)$ then it could be presumed that this drift occurred on Bermuda alone.

The change in frequency of each allele estimates its drift. For allele $j$ at locus $i$ an estimate, $f^{*}{ }_{i j}$, of the effective inbreeding coefficient, $F_{e}$, can be obtained from the relation

$$
f_{i j}^{*}=\frac{\left(P_{i j}(B)-P_{i j}(J)\right)^{2}}{P_{i j}(J)\left(1-P_{i j}(J)\right)}
$$

(Grow and Kimura, 1970). Many estimates of $F_{e}$ can be obtained in this way. In practice, these estimates of inbreeding are inflated by sampling error associated with the sampling of the Jamaica and Bermuda populations. A better estimate is $\hat{f}_{i j}$,

$$
\hat{f}_{i j}=f_{i j}^{*}-\left(\frac{1}{2 M_{J}}+\frac{1}{2 M_{B}}\right),
$$


where $M_{J}$ and $M_{B}$ are the sizes of the samples from the two locations (Krimbas and Tsakas, 1971). These estimates are shown in the table 1.

Invariant loci and rare alleles were excluded from the analysis. This is because larger amounts of genetic change give larger $\hat{f}_{i j}$. Any allele can drift only to fixation or loss. For rare alleles this is only a small amount, so including these in the analysis would lessen the probability of observing large values of $\hat{f}_{i j}$. To avoid such bias only those alleles with frequency greater than 0.05 in the Jamaican population were included.

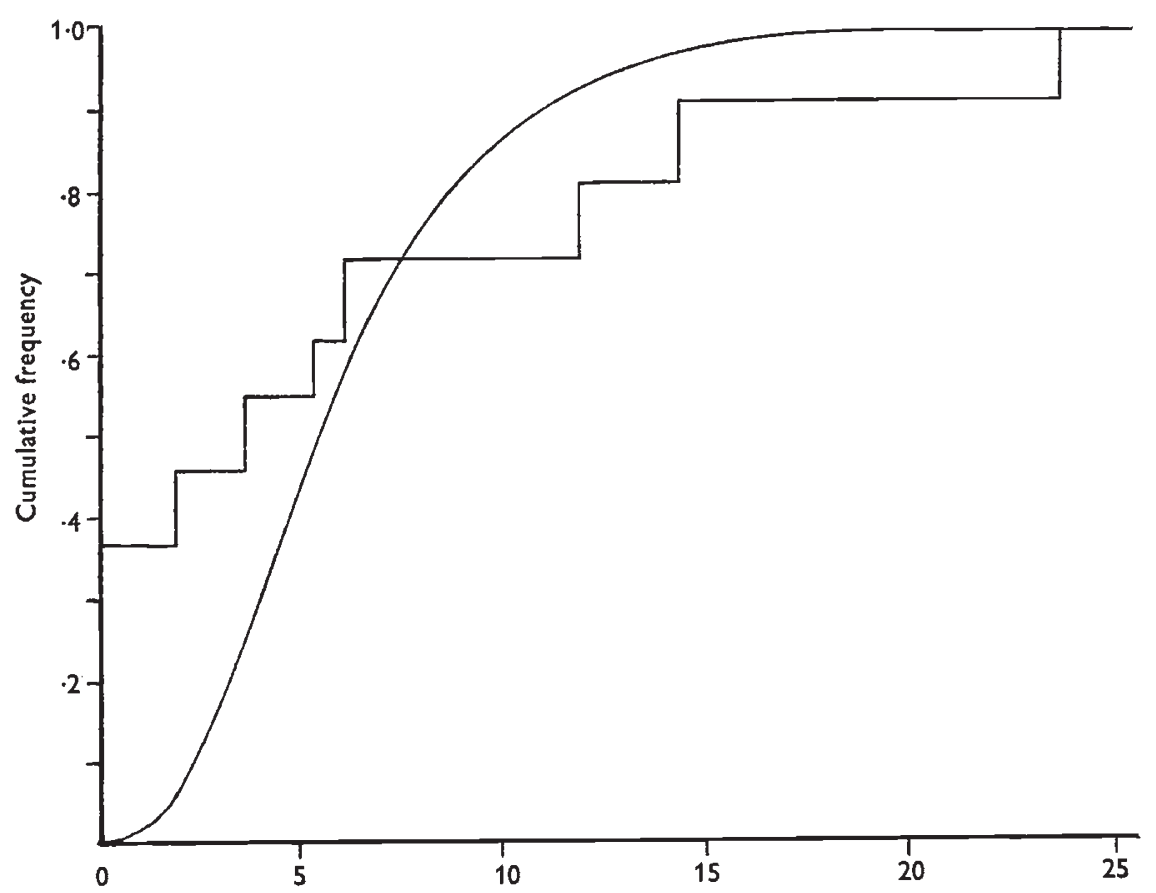

FIG. 1.-Comparison of observed values of $(n-1) \hat{f}_{i j} / F$ (stepped curve) to the $\chi^{2}$ distribution with six degrees of freedom (continuous curve).

Not all of the $\hat{f}_{i j}$ shown in table 1 are independent estimates of the inbreeding coefficients. For example, when there are just two alleles at one locus, as with peptidase, both alleles give the same estimate of $F_{e}$. Lewontin and Krakauer suggest that if there are $k_{i}$ alleles at the $i$ th locus then there are $k_{i}-1$ independent estimates of $F_{e}$ from that locus. The total number of independent estimates is then

$$
n=\sum_{i}\left(k_{i}-1\right)
$$

For the data presented here $n=7$.

Consider one estimate of $F_{e}$ from each locus having $k_{i}=2$ alleles and all $k_{i}$ estimates from loci having more alleles. Let $F$ be their mean. Lewontin and Krakauer (1973) show that $(n-1) \hat{f}_{i j} / F$ is distributed approximately as $\chi^{2}$ with $n-1$ degrees of freedom.

The cumulative frequency distribution of $(n-1) \hat{f}_{i j} / F$ observed for Anolis grahami is plotted in fig. 1 together with the predicted distribution having 
six degrees of freedom. The predicted and observed distributions are apparently different, the variance of the observed distribution being greater than that predicted.

The variance, $\sigma^{2}$, of a $\chi^{2}$ distribution with $n-1$ degrees of freedom is $2(n-1)$, in this case 12 . The observed variance, $s^{2}$, equals 58.56 . The two-sided hypothesis that $s^{2}=\sigma^{2}$ can be tested with an $F$ test having $n-1$ and $\infty$ degrees of freedom (Sokal and Rohlf, 1969) giving, in this case $F=4.88 ; \mathrm{P}<0.001$. The distribution observed here is not in accord with expectations from simple models of neutral alleles. We conclude that not all of the allozymes used in this analysis are neutral.

\section{Discussion}

While not consistent with neutrality at all loci, these results are not necessarily in conflict with the theory of evolution by random walk described by Kimura and Ohta (1971). This theory does not state that all allozymes are neutral, but only that a large portion, say 90 per cent of them are. The number of alleles analysed above is at the lower limit of the effectiveness of this method. As pointed out by Lewontin (1974), many more should normally be used. Yet even if 90 per cent of all allozymes were neutral we might expect at least one non-neutral allele to be among the 12 alleles examined above. With more alleles the inclusion of some which are selected approaches certainty. The conclusion reached here, that not all alleles are neutral, is expected under " neutralist" as well as "selectionist" theory.

Yet the theory of neutral alleles is capable of describing so many situations that exceptions are notable. These results add to the literature that while powerful, the theory of neutral alleles, at least in simple form, does not describe all patterns of allozyme variation (e.g. Dobzhansky, 1970; Allard and Kahler, 1972; Lewontin, 1974).

It may be that the mean value of $\hat{f}_{i j}$ is not far different from $F_{e}$, even if the variance of $\hat{f}_{i j}$ is not in complete accord with neutrality at all loci. This might be expected when most, if not all, allozymes were selectively neutral. The unique history of the Anolis grahami population on Bermuda would then permit an estimate of its effective population size, $\mathcal{N}_{e}$, to be made. Assuming the population behaves "well", the effective inbreeding coefficient $\mathcal{T}$ generations after colonisation is given by the formula

$$
F_{e}(T)=1-\left(1-\frac{1}{2 N_{e}}\right)^{T}
$$

for neutral alleles (Crow and Kimura, 1970). Sixty-eight $=\tau$ years have elapsed between the introduction and sampling of the Bermuda population, and from the preceding analysis $F_{e}(68)=0.0517$. This formula, together with these values, then gives an estimate of $\mathcal{N}_{e}=641$.

This effective population size of Anolis grahami is somewhat larger than that estimated for the rusty lizard, Sceloporus olivaceus $\left(\mathcal{N}_{e}=250\right.$, Kerster, 1964) and much larger than that estimated for the side-blotched lizard, Uta stansburiana $\left(\mathcal{N}_{e}=14\right.$; Tinkle, 1965).

The rate of genetic drift depends on population size-smaller populations drift faster. The population size estimated here does not seem unreasonably 
small, so random genetic drift could occur fast enough to produce the changes observed. We observed, in fact, less genetic change than would be expected by random genetic drift alone in the Uta and Sceloporus populations.

There is no problem believing that random genetic drift could act fast enough to produce the changes we observed. However, we find that the rates of evolution are not homogeneous for the several allozyme alleles examined, varying much more than would be expected if the alleles were all neutral.

Acknowledgments.-We thank Professors Timothy Prout and Richard C. Lewontin for their suggestions and Dr Y. J. Kim for his assistance in running the starch gels. This study was supported by NSF grant GB-40070.

\section{REFERENGES}

Allard, R. W., AND KAhler, A. L. 1972. Patterns of molecular variation in plant populations. In L. Le Cam, J. Neyman, and E. L. Scott (Eds.), Proceedings of the Sixth Berkeley Symposium on Mathematical Statistics and Probability. Vol. 5, Darwinian, Neo-Darwinian and Non-Darwinian Evolution. University of California Press, Berkeley.

dobzhansky, Th. 1970. Genetics of the Evolutionary Process. Columbia University Press, New York.

GROW, J. F., AND KIMURA, M. 1970. An Introduction to Population Genetics Theory. Harper and Row, New York.

Fox, w. 1963. Special tubules for sperm storage in female lizards. Nature, 198, 500-501. GORMAN, G. G., SOULE, M., YANG, s. Y., AND NEVO, E. 1975. Evolutionary genetics of insular Adriatic lizards. Evolution. (In press.)

KERSTER, w. 1964. Neighbourhood size in the rusty lizard, Sceloporus olivaceus. Evolution, $18,445-457$.

kImURA, M., AND ohta, T. 1971. Theoretical Aspects of Population Genetics. Princeton University Press, Princeton, New Jersey.

KRIMBAS, C. B., AND TSAKAs, s. 1971. The genetics of Dacus oleae. V. Changes of esterase polymorphism in a natural population following insecticide control. Selection or drift? Evolution, 25, 454-462.

LEWontin, R. G. 1974. The Genetic Basis of Evolutionary Change. Columbia University Press, New York.

LEWONTIN, R. C., AND KRAKAUER, J. 1973. Distribution of gene frequency as a test of the theory of the selective neutrality of polymorphisms. Genetics, 74, 175-195.

LICHT, P., AND GORMAN, G. c. 1970. Reproductive and fat cycles in Caribbean Anolis lizards. Univ. Calif. Publ. Zool., 95, 1-52.

NEvo, E. 1973. Test of selection and neutrality in natural populations. Nature, 244, 573-575.

SOKAL, R. R., AND ROHLF, F. J. 1969. Biometry. Freeman, San Francisco.

TINKLE, D. W. 1965. Population structure and effective size of a lizard population. Evolution, 19, 569-573.

WINGATE, D. B. 1965 . Terrestrial herpetofauna of Bermuda. Herpetologica, 21, 202-218.

YANG, S. Y., SOULE, M., AND GORMAN, G. G. 1974. Lizards of the eastern Caribbean: A case study in evolution. I. Genetic relationships, phylogeny, and colonization sequence of the roquet group. Systematic Zoology, 23, 387-399. 